\title{
ON THE EFFECT OF SHAPE PARAMETERIZATION ON AEROFOIL SHAPE OPTIMIZATION
}

\author{
Kailash Manohara Selvan ${ }^{1}$ \\ ${ }^{1}$ Formerly at Hindustan University, Department of Aeronautical Engineering, Chennai, India
}

\begin{abstract}
The selection of suitable shape parameterization technique is one of the significant factors affecting the fidelity of the solution found during aerofoil shape optimization process. This paper investigates the effect of shape parameterization on an automated aerofoil shape optimization problem. Four well known shape parameterization techniques were considered for study; Bezier curves, Class-Shape function Transformation, Hicks-Henne "Bump" function and polynomial method. A boundary layer panel code was coupled with a surrogate-based multi-objective evolutionary algorithm and implemented within an automatic design loop. The optimization problem was formulated for NACA 0012 aerofoil at 5 degree angle of attack. The main criteria for comparison were based on the number of parameters required by each method for accurate representation of the aerofoil, the ability to find the aerofoil with the best performance within the constrained design space and also the computational cost. Preliminary results show that the optimization process was able to increase the lift-to-drag ratio of the aerofoil by $30 \%$. ClassShape Transformation and Hicks-Henne Bump function were able to find the best aerofoil shape within the design space effectively.
\end{abstract}

Keywords-Aerofoil Optimization, Evolutionary Algorithm, Shape Parameterization

\section{INTRODUCTION}

In the recent years, aerodynamic shape optimization has attracted extensive research interest. Mathematically representing the aerofoil shape during the optimization process is a difficult problem. Shape parameterization deals with representing the aerofoil shape in terms of influential design parameters. There exist many techniques for parameterization, each requiring different number of design parameters to represent an aerofoil. More the number of parameters, the optimization process could be capable to find novel aerofoil shapes. On the other hand, with more design parameters, it would be expensive to search the design space. In addition, the interaction between the parameters may lead to un-realistic aerofoil being produced [1].

Aerofoils can be represented as coordinates points. This method can be capable to represent a variety of aerofoils and also reflect precise local changes. However, it would be very difficult to use coordinate based method within an optimization process, because it would involve enormous number of parameters and the associated high computation cost in exploring the design space. Another method of representing the aerofoil is in the form of B-Splines. Some of the disadvantages of B-Spline method are presented in [1]. PARSEC technique is another popular parameterization technique. The comparison of coordinate method, B-Spline curves and PARSEC method for optimization and inverse design problem is presented in [1],[2] and [3].

This paper studies the effect of four shape parameterization methods on optimization of a subsonic aerofoil. The methods studied are Bezier curves, Class-Shape function
Transformation (CST), Hicks-Henne "Bump" (HHB) function and Polynomial method. The paper is organized as follows; the different parameterization techniques are described in the next section, which is followed by the description of the framework that was developed for the research. The problem setup and the results are presented and discussed in the subsequent sections.

\section{AEROFOIL SHAPE PARAMETERIZATION}

\subsection{Class-Shape function Transformation (CST)}

\section{Technique}

This technique proposed by Kulfan and Bussoletti [4] represents the shape of a two-dimensional aerofoil in terms of a shape function $S(x)$, class function $C(x)$ and trailing edge thickness $\Delta z_{t e}$.

$$
y=C(x) \cdot S(x)+x \Delta z_{t e}, 0 \leq x \leq 1
$$

The class function $C(x)$ is given by,

$$
C(x)=x^{N_{1}}[1-x]^{N_{2}}
$$

Where $N_{1}$ and $N_{2}$ define a specific aerofoil class. Different aerofoil class exists including elliptical $\left(N_{1}=N_{2}=0.5\right)$, Sears-Haack body $\left(N_{1}=N_{2}=0.75\right)$, biconvex $\left(N_{1}=N_{2}=\right.$ 1) etc. For NACA type aerofoils with rounded leading edge and blunt trailing edge $N_{1}=0.5$ and $N_{2}=1$.

In principle, the shape function can be arbitrary. However, it is convenient to choose a family of well behaved analytical functions, to generate $\mathrm{S}(\mathrm{x})$, so that the class function $\mathrm{C}(\mathrm{x})$, 
should remain as the only source of non-analyticity of the representation [4]. A weighted sum of Bernstein polynomials can be used as shape function, Eq (7)

$$
S(x)=\sum_{i=1}^{i=n} b_{i} \cdot K_{i} \cdot x^{i} \cdot(1-x)^{i}
$$

For $\mathrm{N}_{1}=0.5$ and $\mathrm{N}_{2}=1$, the shape function at the extremes can be related to leading edge radius $\left(\mathrm{R}_{\mathrm{LE}}\right)$ and trailing edge thickness $\left(\Delta \mathrm{z}_{\mathrm{te}}\right)$ and angle $(\beta)$ as follows,

$$
S(0)=\sqrt{R_{L E}} ; S(1)=\tan \beta+\Delta z_{t e}
$$

Fig 1 shows the parametric aerofoil generated using CST technique; (a) shows the Class function with $N_{1}=0.5$ and $N_{2}=1$, (b) shows the Shape function with $1^{\text {st }}$ order and $3^{\text {rd }}$ order Bernstein polynomials and (c) shows the parametric aerofoil.

\subsection{Hicks-Henne "Bump" Function}

This technique proposed by Hicks and Henne [5][6], uses a set of smooth function to perturb the initial aerofoil. The aerofoil is represented as a weighted sum of sine function.

$$
\begin{gathered}
y=y_{\text {basis }}+\sum_{i=1}^{i=n} \alpha_{i} f_{i}(x) \\
f_{i}(x)=\left[\sin \left(\pi x^{\frac{\log \frac{1}{2}}{\log t_{1}}}\right)\right]^{t_{2}}, 0 \leq x \leq 1
\end{gathered}
$$

$t_{1}$ and $t_{2}$ define the maximum thickness location of the bump and the width of the bump respectively. $\alpha_{i}$ denote the shape parameters. An important advantage of this method is that it allows specific region of the aerofoil to be perturbed while maintaining the others virtually undisturbed.

Fig 2 shows an aerofoil represented through HHB technique with 2 sine functions $\left(\mathbf{t}_{\mathbf{1}}=\mathbf{0 . 3 3} \& 0.661\right)$

\subsection{Bezier}

A Bezier curve is a parametrically defined curve given by,

$$
P(t)=\sum_{i=1}^{i=n} B_{i, n} . P_{i}
$$

$P$ is the vector of control points of the parametric curve $P(t)$. The blending function $B_{i, n}$ is the Bernstein polynomial given by,

$$
\begin{gathered}
B_{i, n}=K_{i} \cdot t^{i} \cdot(1-t)^{i}, 0 \leq t \leq 1 \\
K_{i}=\frac{n !}{n ! \cdot(n-i) !}
\end{gathered}
$$

The Bezier curve can be used to parameterize any arbitrary curve and therefore they can be used for a variety of applications including aerofoil optimization.

Fig 6 shows an aerofoil represented using a Bezier curve with 6 control points.

\subsection{Polynomial Function}

The aerofoil shape can be represented by higher order polynomials, Eq (9).

$$
y=y_{\text {basis }}+\sum_{i=1}^{i=n} \alpha_{i} x^{n}, 0 \leq x \leq 1
$$

The number of parameters is defined by the order of the polynomial chosen. PARSEC method [7] is a kind of polynomial model which uses 11 parameters to represent the aerofoil.

$$
y_{P A R S E C}=\sum_{i=1}^{i=6} \alpha_{i} x^{i-\frac{1}{2}}
$$

However, in this paper a generic polynomial function is considered.

A simulation framework Project X-2D was developed as part of this research. A brief description about the framework is presented in the next section.

\section{PROJECT X - 2D}

Project-X 2D comprises a set of program codes specifically developed for automatic design and optimization of twodimensional aerofoils. The illustration of Project-X 2D framework is presented in Fig 5.

The user specifies the reference aerofoil. The reference aerofoil is parameterized by the Para_X module by one of the techniques described in the previous section. The user then defines the design space by specifying the bounds/constraints for each shape parameter.

Conventionally, aerofoils will be generated by randomly picking parameters from the defined design space. In the present work, aerofoils are not generated randomly; rather, they are generated using parameters selected by Design of Experiments (DoE) technique. Latin-Hypercube Sampling (LHS) technique is implemented within this framework. LHS uses a stratified sampling scheme to improve on the coverage of the input design space. An array of aerofoil shapes are generated and stored by the DoE_X module.

The generated aerofoils are solved by Xfoil [8]. XFOIL is a design and analysis system for isolated subcritical airfoils. A linear-vorticity second order accurate panel method is used in the inviscid flow. This method is coupled with an integral boundary-layer method and an $e^{n}$-type transition amplification formulation using a global Newton method.

XFOIL was chosen for use in Project X-2D based on its speed and accuracy. It is very fast compared with Reynoldsaveraged Navier-Stokes (RANS) methods, and it has been proven to be well suited for the analysis of subsonic airfoils even at low Reynolds numbers [9]. 


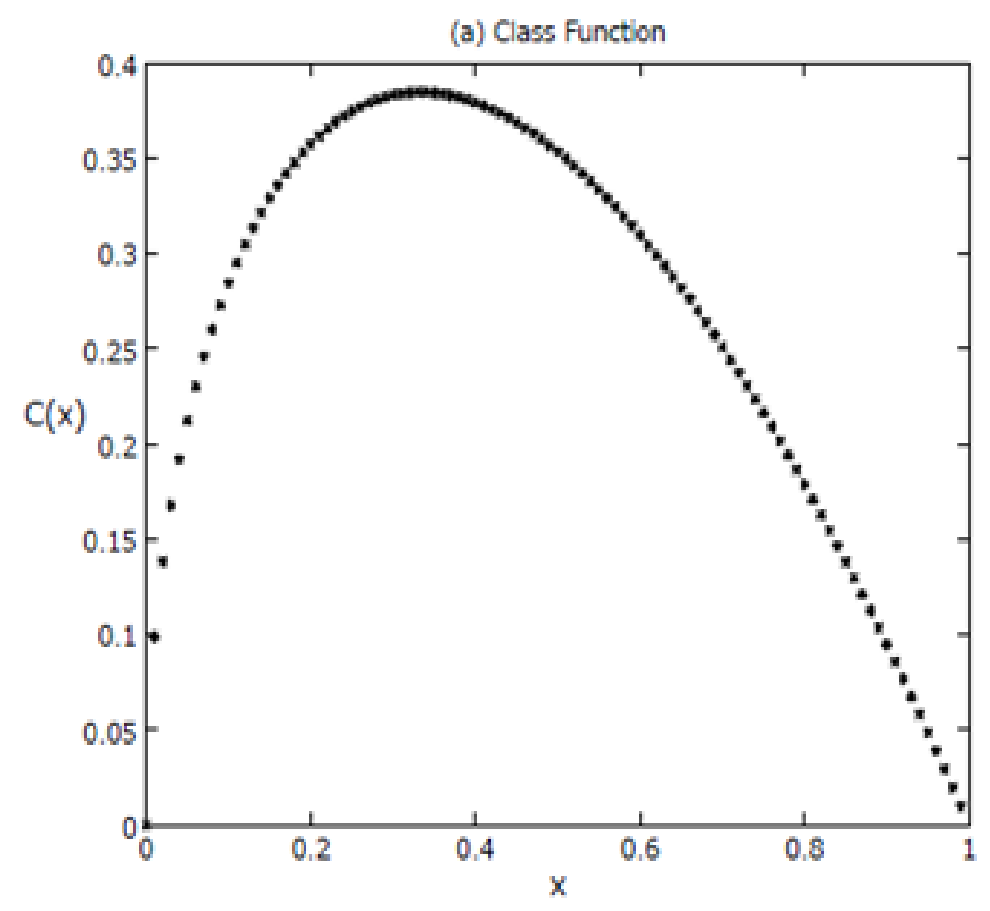

(b) Shape Function

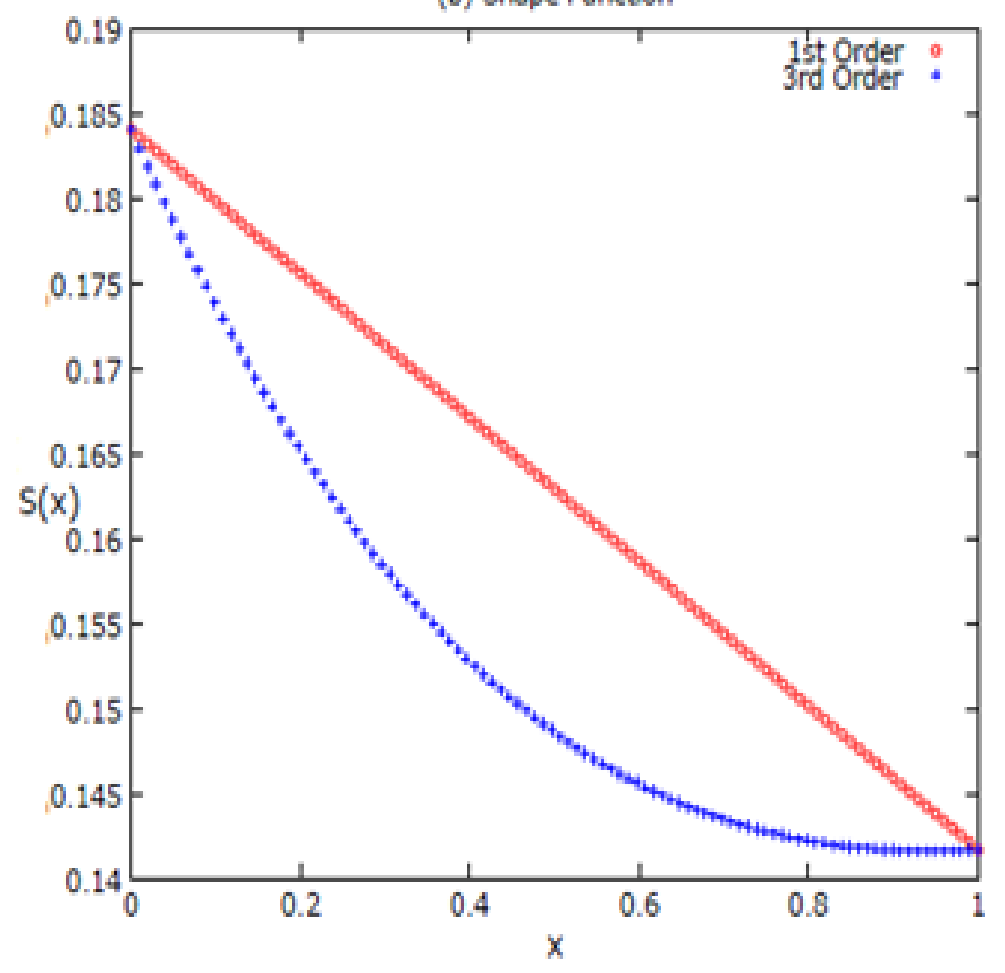


(c) Parametric Aerfoil (CST)

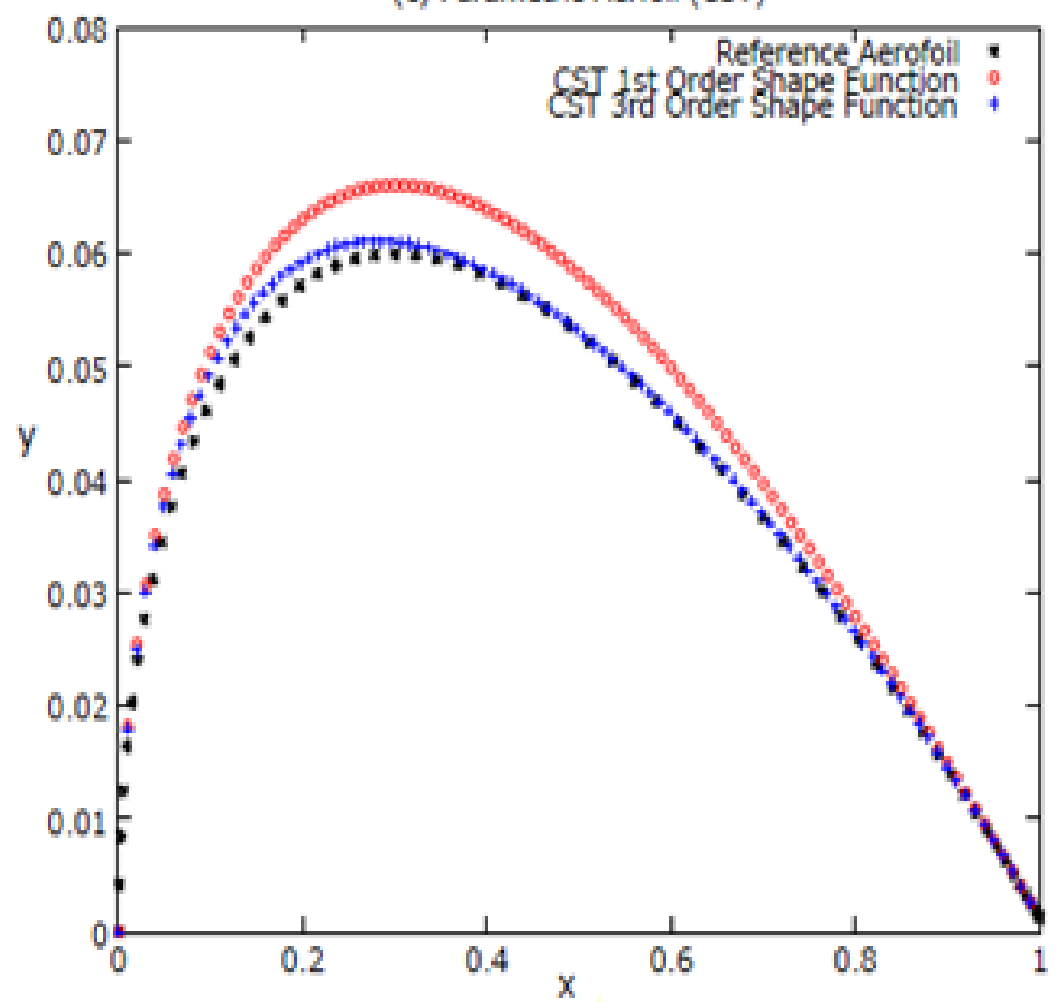

Fig 1 Shape parameterization using CST technique with $1^{\text {st }}$ order and $3^{\text {rd }}$ order shape function

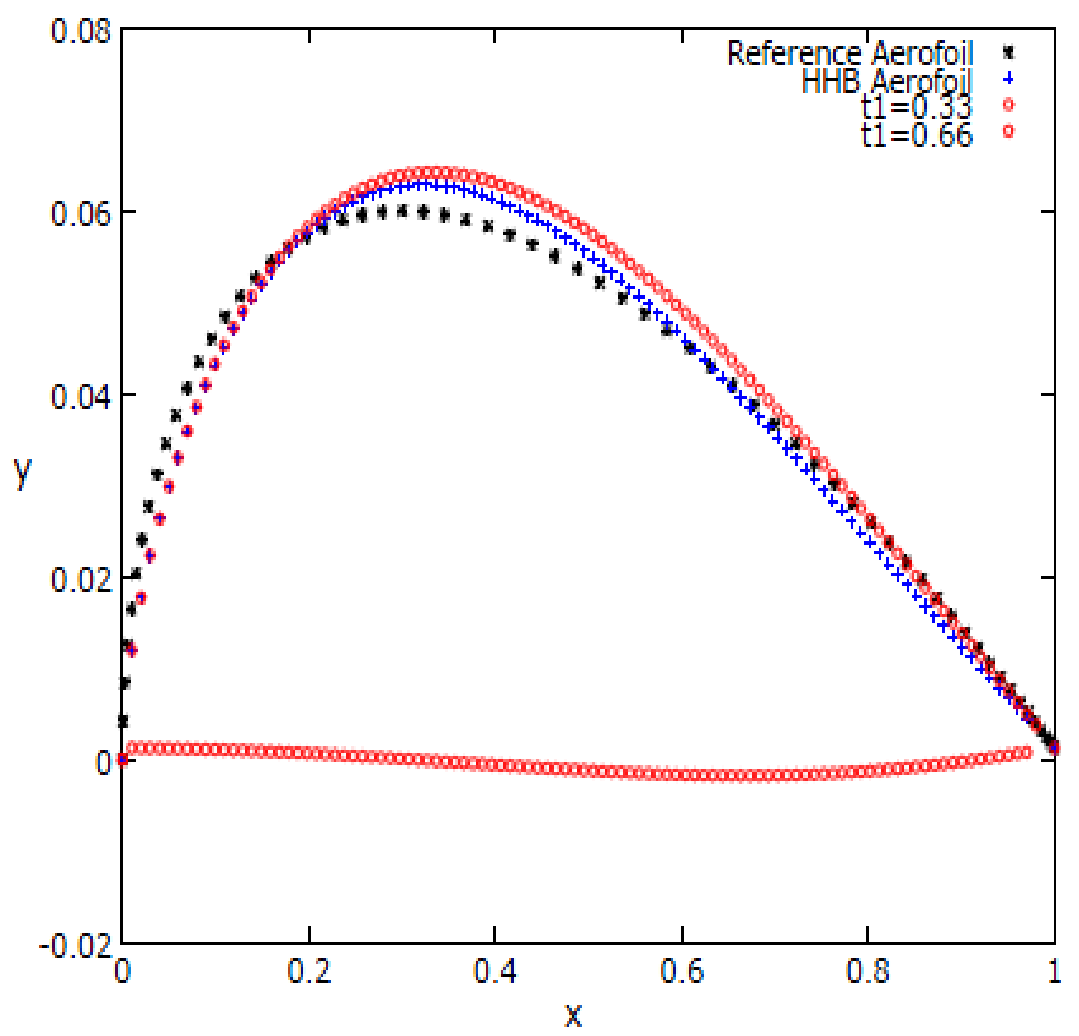

Fig 2 Shape parameterization using HHB technique $\left(t_{2}=\mathbf{1}\right.$ and $\boldsymbol{t}_{\mathbf{1}}=\mathbf{0 .} 33$ \& 0.661) 


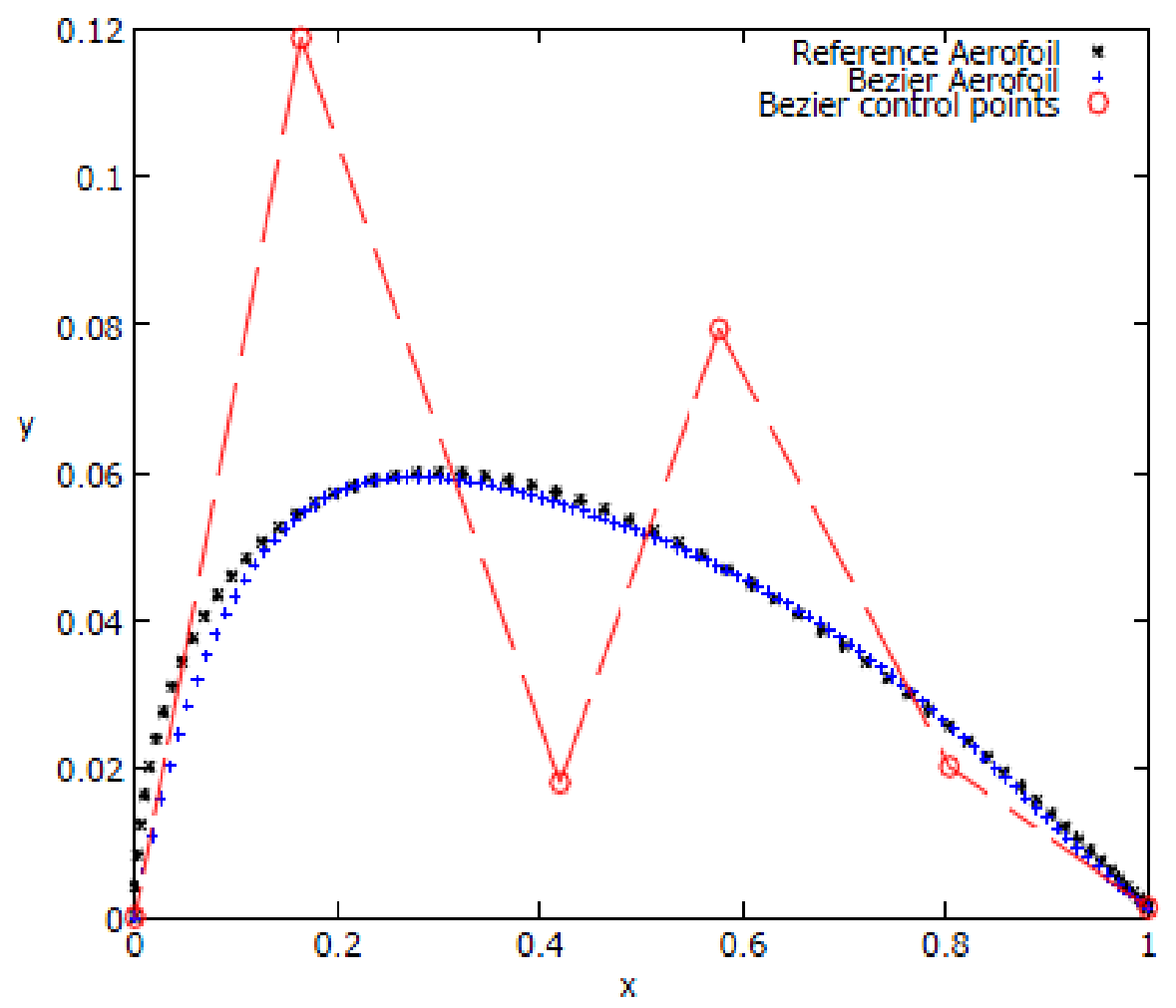

Fig 3 Shape parameterization using Bezier technique with 6 control points

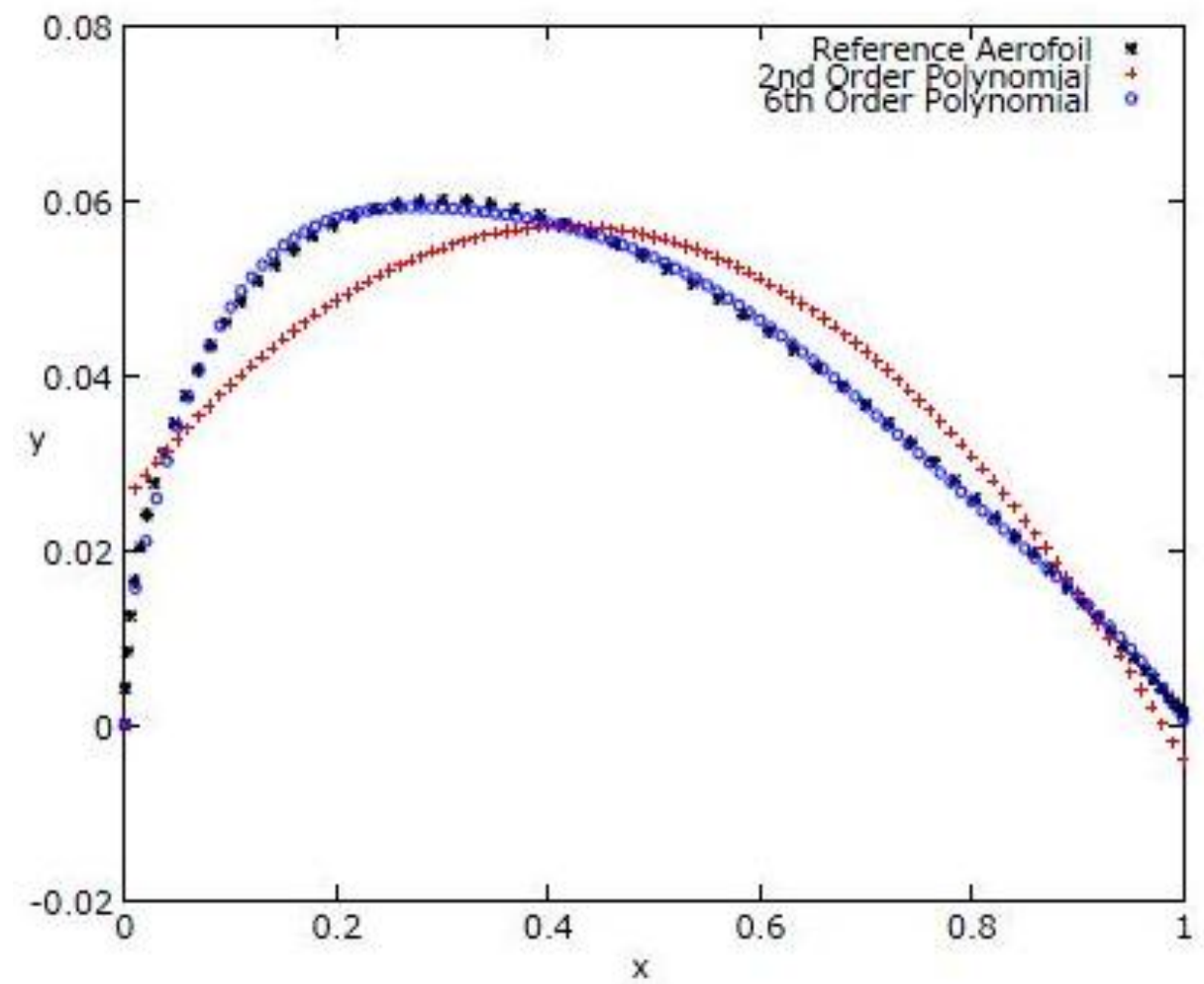

Fig 4 Shape parameterization using Polynomial technique ( $2^{\text {nd }}$ order and $6^{\text {th }}$ order $)$ 


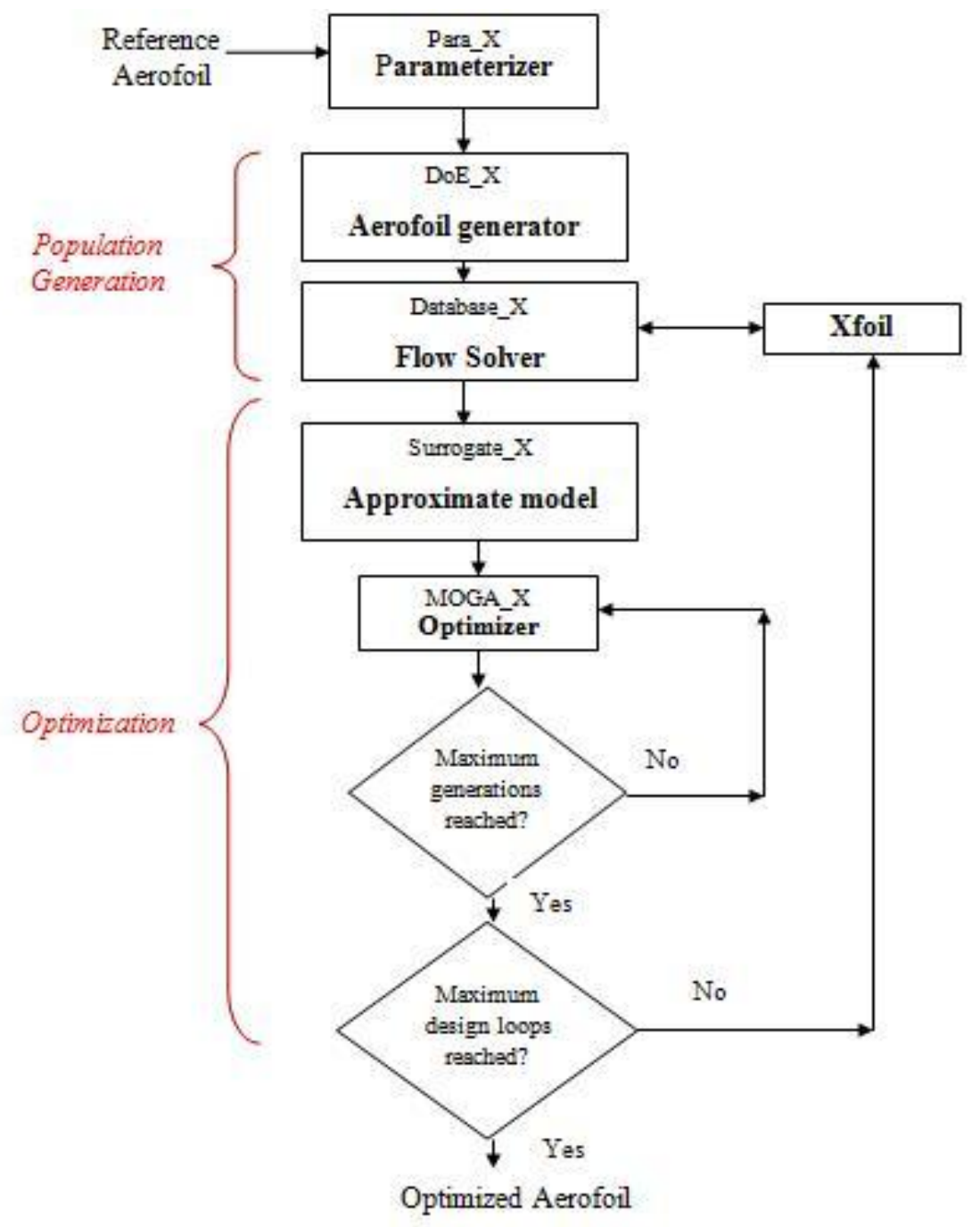

Fig 5 Project-X 2D Framework

Xfoil provides the aerodynamic performance (lift and drag coefficients) for each aerofoil generated by DoE_X module. The design parameters for the initial population and their corresponding aerodynamic data are stored as a database by Database_X module.

A surrogate (approximate) model can be constructed which closely mimics the relationship between the input geometric parameters and output performance variables. This approximate model can be used during the optimization process. The total number of flow solver calls can be noticeably reduced through this technique, which in turn reduces the total time without deteriorating the performance of the optimization process [10]. Response surface approximations (RSA) are often used as inexpensive replacements for computationally expensive computer simulations. Once a RSA has been computed, it is cheap to evaluate the objective functions during optimization. So a RSA based surrogate model is implemented within this framework.
Evolutionary Algorithm (EA) or Genetic Algorithm (GA) is very popular for engineering design optimization. They were originally developed using natural evolution analogy. They facilitate the set of designs "offsprings" with improved performance or "fitness" to be evolved, through GA operators; crossover, mutation and selection of the best designs from the previous generation of. designs "parents". This algorithm is renowned for its ability to seek the global optimum and the ability to handle multiple objectives effectively. A Multi-Objective Genetic Algorithm, MOGA_X module was developed and implemented within this framework.

The MOGA_X module uses the approximate model during design space exploration. The optimizer performs a predefined number of generations and the flow solver is called only for the optimal design found by this inner optimization loop. This design is added into the database with an intention to improve the accuracy of the approximate model for the next design/optimization cycle. The robustness of this methodology is affected by the stability of the surrogate model and the construction of a 
stable approximate model is essential. This methodology is expected to converge much faster than conventional optimization methods. A predefined number of design iterations are performed before producing the optimal aerofoil shape.

The problem setup and the results are presented in the next section.

\section{RESULTS}

\subsection{Problem Setup}

Any optimization problem needs initial reference geometry to start with. In the present study, NACA 0012 aerofoil was specified as the reference. The next step is specifying the bounds through which the aerofoil shape could be perturbed during the optimization process. Mechanical, structural considerations might limit the design space. In this case the design space was constrained within the minimum and maximum bounds specified. The bounds and the reference aerofoil are shown in Fig 6.

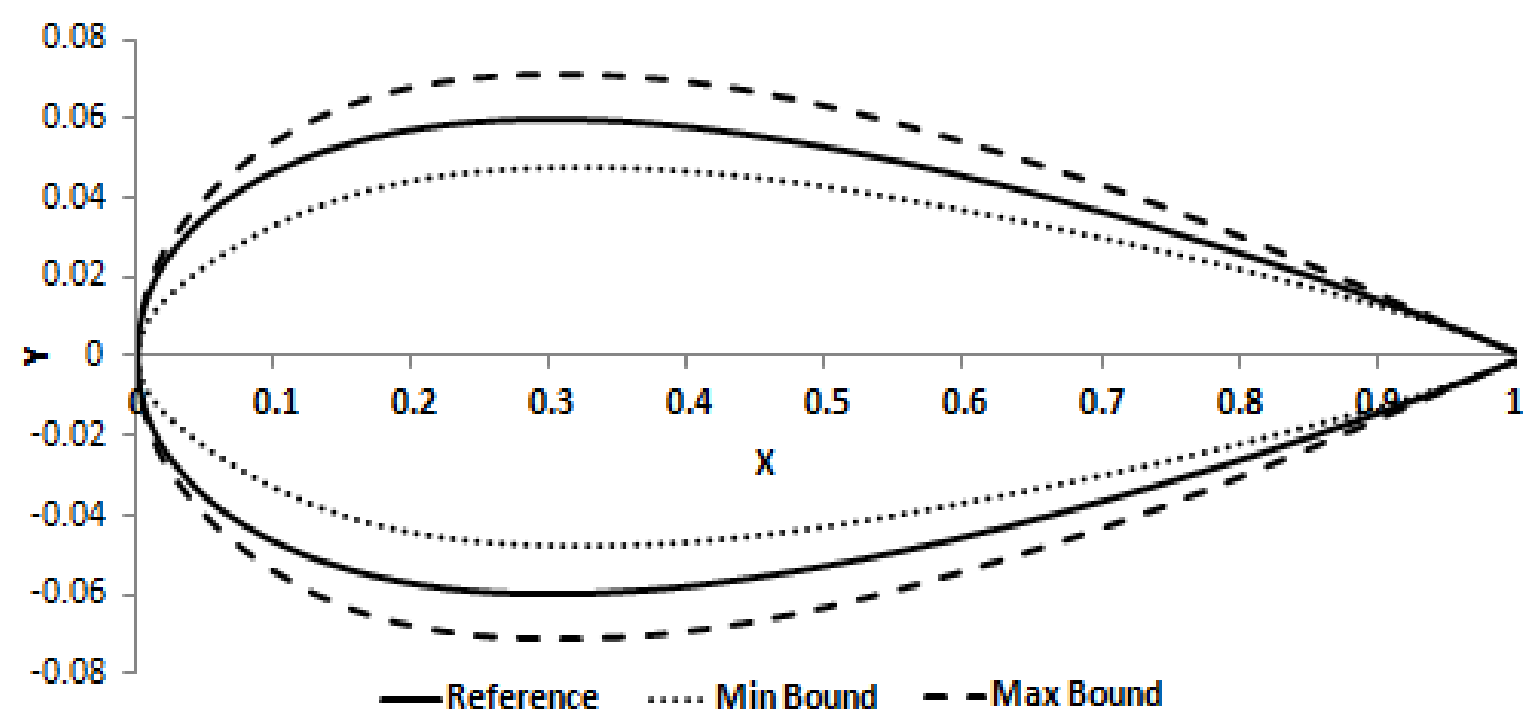

Fig 6 Reference Aerofoil (NACA 0012) with minimum and maximum bounds specified

Different parameterization techniques were used to parameterize the reference aerofoil. The suction and pressure surfaces were parameterized separately. LHS method was used to select the initial population from the design space. The effect of the initial sample size using LHS on the accuracy of the response surface constructed was investigated in [11]. They confirmed that the initial sample size had very little effect on the accuracy of the response surface model constructed using LHS method. Initial population size was selected as 2-3 times the number of the parameters in each case. The initial population of aerofoils was solved using Xfoil to obtain the aerodynamic performance characteristics.

The flow conditions used by the solver are presented below in Table 1.

Table 1 Inlet flow conditions

\begin{tabular}{|l|c|}
\hline Flow conditions \\
\hline Mach Number & 0.2 \\
\hline Reynolds Number & $1 \times 10^{6}$ \\
\hline Angle of attack & $5^{0}$ \\
\hline
\end{tabular}

A response surface approximation was constructed on this data. This model was used by the optimizer for objective function evaluations within the inner optimization loop. The flow solution is computed only on the final shape found at the end of a design loop. This new flow solution is added into the database and used for constructing the response surface model for the next design loop. 20 generations were allowed within each inner optimization loop and 20 design cycles were carried out. All simulations were carried out on an Intel dual core machine with 2GB RAM.

\subsection{Lift Maximization}

An optimization problem can have many objectives. Usually, the multiple objectives can be reduced to a single objective. This is done by assigning them some weighting factors, and then use the single objective during optimization.

The goal of this optimization problem was to find the aerofoil shape from the constrained design space with the same drag coefficient as the reference aerofoil but with higher lift coefficient. The lift and drag coefficients of the 
reference aerofoil for the flow conditions shown in Table 1 were, $C_{L_{\text {Reference }}}=0.56$ and $C_{D_{\text {Reference }}}=0.0086$. The objective function formulated for this optimization problem is given in $\mathrm{Eq}(11)$.

$$
\begin{gathered}
\text { Objective Function } \\
I=\sqrt{w_{1}\left(\frac{\left.C_{L_{\text {Reference }}}\right)^{2}+w_{2}\left(\frac{C_{D^{-}-C_{D} \text { Reference }}}{C_{L}}\right)^{2}}{C_{D}}\right.} \\
w_{1}=1 w_{2}=10
\end{gathered}
$$

\subsection{Accuracy}

The reference aerofoil was parameterized using all the four techniques. The number of parameters required by each of these techniques to adequately represent the suction surface of the aerofoil was studied. The error in parameterization was computed by Eq (12). Parameterizations with error less than $5 \times 10^{-4}$ were accepted.

$$
\text { Error }=\frac{\left|\int y_{\text {reference }} d x-\int y_{\text {parametric }} d x\right|}{\int y_{\text {reference }} d x}
$$

Fig 7 presents the change in error with the number of parameters for each parameterization technique. It shows that, HHB and CST technique require the least number of parameters; they require 4 parameters to adequately represent the suction surface of the reference aerofoil, while polynomial and Bezier techniques require 7 and 12 parameters respectively. Twice as much parameters were required to represent the complete aerofoil.

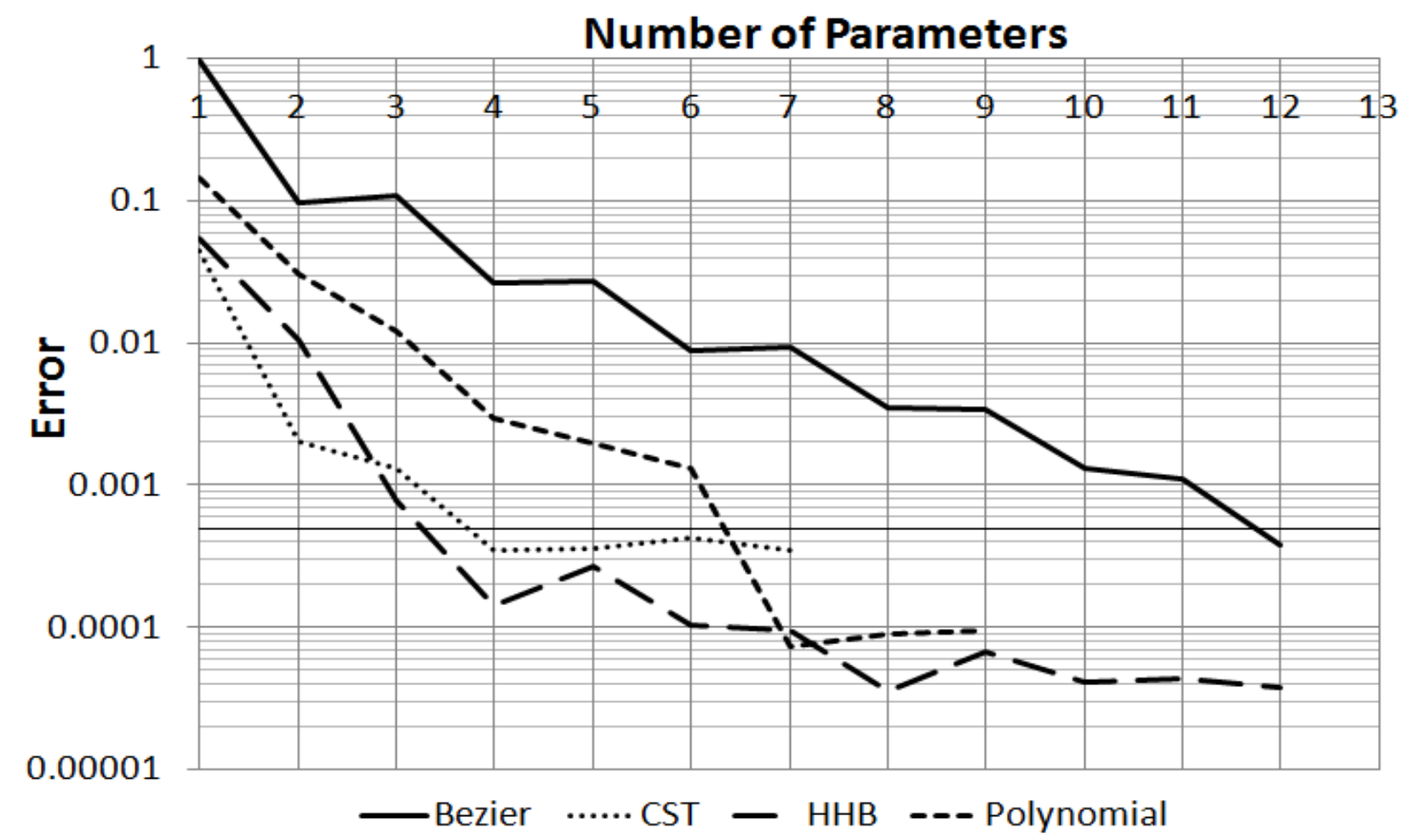

Fig 7 Comparison of number of parameters and error associated with different parameterization techniques

Table 2 Number of parameters required by each parameterization technique to represent the complete aerofoil

\begin{tabular}{|l|l|}
\hline Cases & Number of parameters \\
\hline Class-Shape function & 8 \\
\hline Hicks-Henne function & 8 \\
\hline Polynomial & 14 \\
\hline Bezier & 24 \\
\hline
\end{tabular}

\subsection{Computational Cost}

More parameters during parameterization imply a bigger initial population. It is obvious that the computation time for initial database generation increases linearly with the number of parameters. In addition to this, the number of parameters also influence the time for approximate model construction and optimization. The time required per design cycle when using different parameterization techniques are presented in Table 3.

Table 3 Comparison of time per design loop for different parameterization techniques

\begin{tabular}{|l|l|}
\hline Cases & $\begin{array}{l}\text { Time per design } \\
\text { loop }[\mathbf{s}]\end{array}$ \\
\hline Class-Shape function & 97 \\
\hline Hicks-Henne function & 92 \\
\hline Polynomial & 148 \\
\hline Bezier & 210 \\
\hline
\end{tabular}




\subsection{Optimum Designs}

The lift coefficients and the lift-to-drag $\left(\boldsymbol{C}_{\boldsymbol{L}} / \boldsymbol{C}_{\boldsymbol{D}}\right)$ ratio of the optimum aerofoils found by the optimizer through different parameterization techniques are presented in Table 4. It can be seen that the HHB and CST parameterization technique was able to improve the lift-to-drag ratio considerably compared to Bezier and polynomial methods. The optimum aerofoil shapes and the corresponding coefficient of pressure (Cp) distribution are presented in Fig 8 to Fig 11. The optimum aerofoils found by HHB and CST techniques have more negative pressure on the suction side and more positive pressure on the pressure side which are key for higher lift. Clearly, for the same design space and with the same optimization strategy, HHB and CST facilitate aerofoils with better aerodynamic performance to be found.

Table 4 Comparison of the performance of optimum aerofoils found through different parameterization techniques

\begin{tabular}{|l|c|c|c|}
\hline Cases & $\boldsymbol{C}_{\boldsymbol{L}}$ & $\frac{\boldsymbol{C}_{\boldsymbol{L}}}{\boldsymbol{C}_{\boldsymbol{D}}}$ & $\Delta\left[\frac{\boldsymbol{C}_{\boldsymbol{L}}}{\boldsymbol{C}_{\boldsymbol{D}}}\right] \%$ \\
\hline Class-Shape function & 0.7087 & 82.407 & 26.55357 \\
\hline Hicks-Henne function & 0.7272 & 84.558 & 29.85714 \\
\hline Bezier & 0.6352 & 73.860 & 13.42857 \\
\hline Polynomial & 0.6263 & 72.441 & 11.83929 \\
\hline
\end{tabular}

\section{CONCLUSION}

Four shape parameterization techniques; Class-Shape function Transformation, Hicks-Henne "Bumps" function, Bezier and polynomial method, are compared for the aerodynamic design and optimization of aerofoils. A framework is developed to facilitate this study. The lift maximization optimization problem was formulated on NACA 0012 reference aerofoil at 5 degree angle of attack. Many important observations were made. Firstly, HHB and CST technique required only 8 parameters to accurately represent the aerofoil. Secondly, HHB and CST techniques with lesser parameters needed atleast $50 \%$ lesser computational time compared to the other techniques. Thirdly, the optimum aerofoil found through CST and HHB aerofoil showed better aerodynamic performance, confirming their better exploratory characteristics. The polynomial method, even if it was able to represent the aerofoil with fewer parameters compared to the Bezier was not able to provide the best aerofoil. It can be concluded that CST to HHB techniques could be very effective in aerofoil shape optimization compared to conventional techniques. The frame work developed could be easily extended to 3D wing design and optimization. The future work would involve the investigation of these parameterization techniques on an inverse design problem.

\section{REFERENCES}

[1] A.Shahrokhi and A.Jahangirian, "The effects of shape parameterization on the efficiency of evolutionary design optimization for viscous transonic airfoils," Journal of Aerospace Science and Technology, pp. Vol. 5, No. 1, pp 35-43, March 2008.
[2] J.Hajek, "Parameterization of Airfoils and Its Application in Aerodynamic Optimization," in Proceedings of WDS'07, Charles University, Faculty of Mathematics and Physics, Prague, Czech Republic, 2007.

[3] P.Castonguay and S.K.Nadarajah, "Effect of shape parameterization on aerodynamic shape optimization," in Proceedings of the 45th Aerospace Sciences Meeting and Exhibit (AIAA '07), pp. 561580, January 2007.

[4] B. Kulfan and J. Bussoletti, "Fundamental Parametric Geometry Representations for Aircraft Component," in 11th AIAA/ISSMO Multidisciplinary Analysis and Optimization Conference, 2006.

[5] R. M. Hicks, E. M. Murman and G. N. Vanderplaats, "An assessment of airfoil design by numerical optimization," in NASA TM X-3092, Ames Research Center, Moffett Field, California, July 1974.

[6] R. M. Hicks and P. A. Henne., "Wing design by numerical optimization," Journal of Aircraft, vol. 15, p. 407-412, 1978.

[7] H.Sobieczky, "Parametric airfoils and wings," Notes on Numerical Fluid Mechanics, p. pages 71-88, 1998.

[8] M.Drela, "XFOIL: An Analysis and Design System for Low Reynolds Number Airfoils," in Low Reynolds Number Aerodynamics, ed. by T. J. Mueller, Vol. 54 of Lecture Notes in Engineering, New York, Springer-Verlag, June 1989, p. p. 1-12

[9] B. A. Gardner and Michael.S.Selig, "Aerofoil design using a genetic algorithm and an inverse method," in 41st Aerospace Sciences Meeting and Exhibit, Reno, Nevada, 6-9 January 2003.

[10] L.P.Swiler, R.Slepoy and A.A.Giunta, "Evaluation of Sampling Methods in Constructing Response Surface Approximations," Sandia National Laboratories, Albuquerque, NM. 

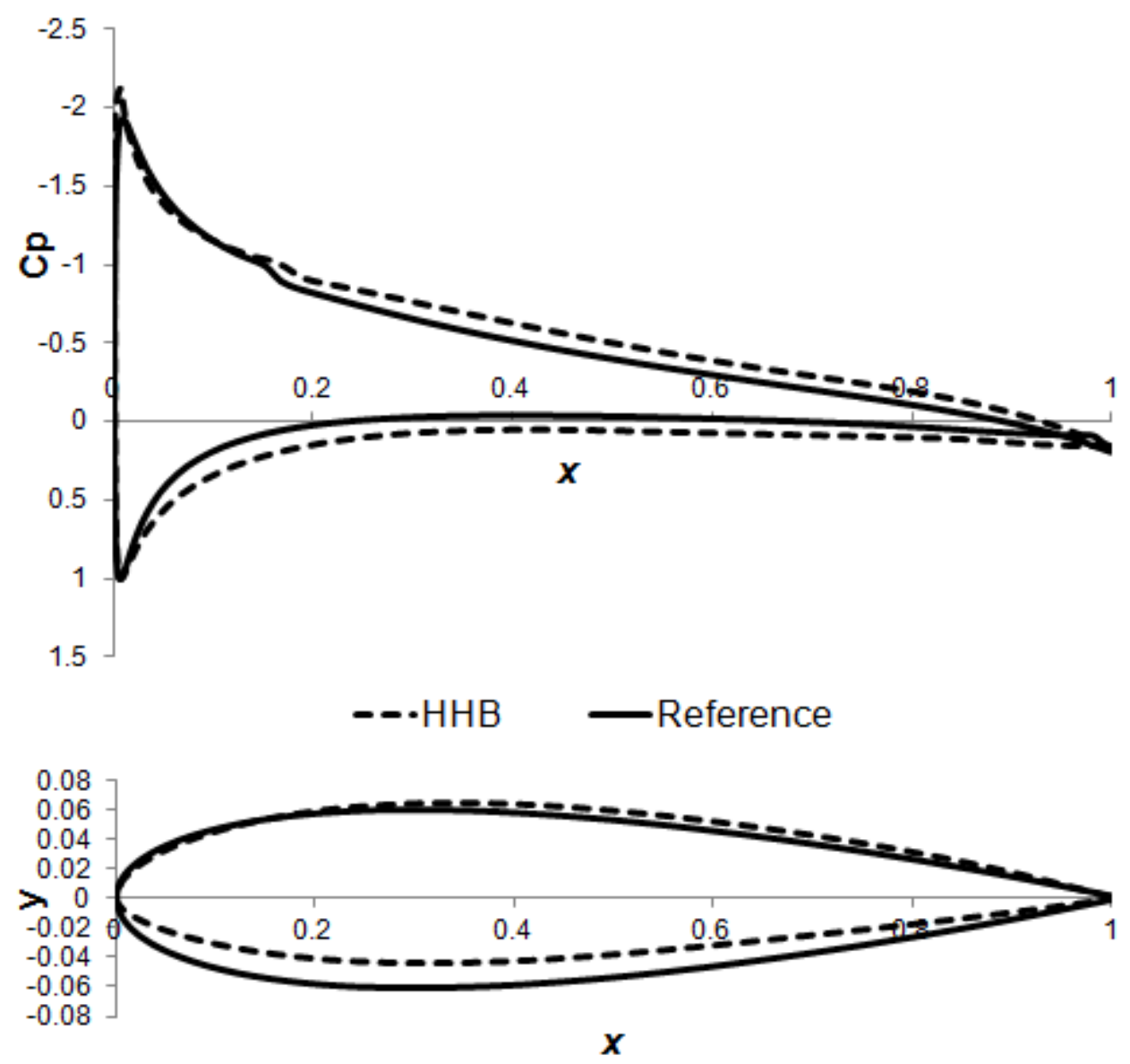

Fig 8 Optimum aerofoil geometry found through HHB parameterization technique and corresponding Cp distribution compared with the reference
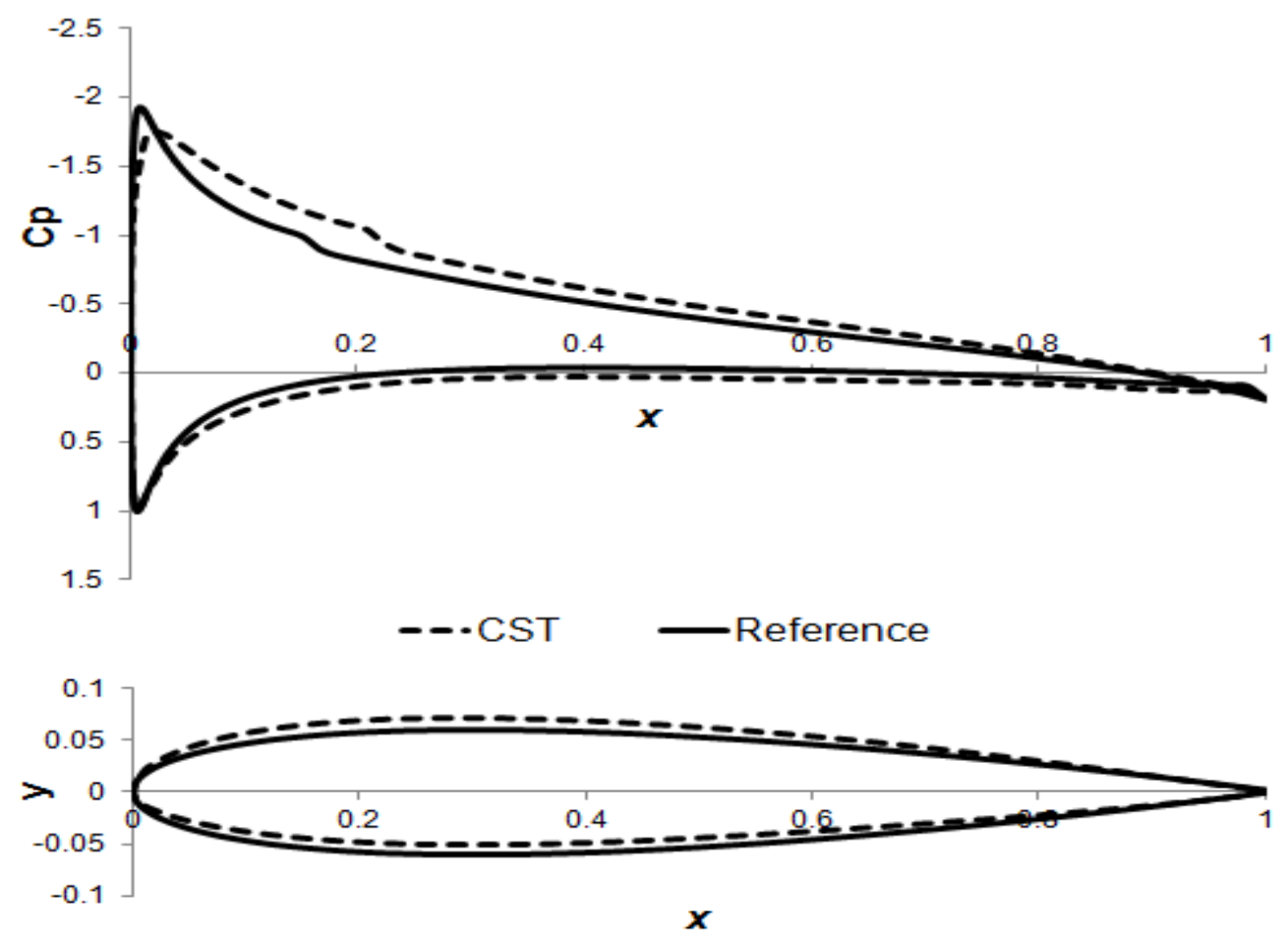

Fig 9 Optimum aerofoil geometry found through CST parameterization technique and corresponding Cp distribution compared with the reference 

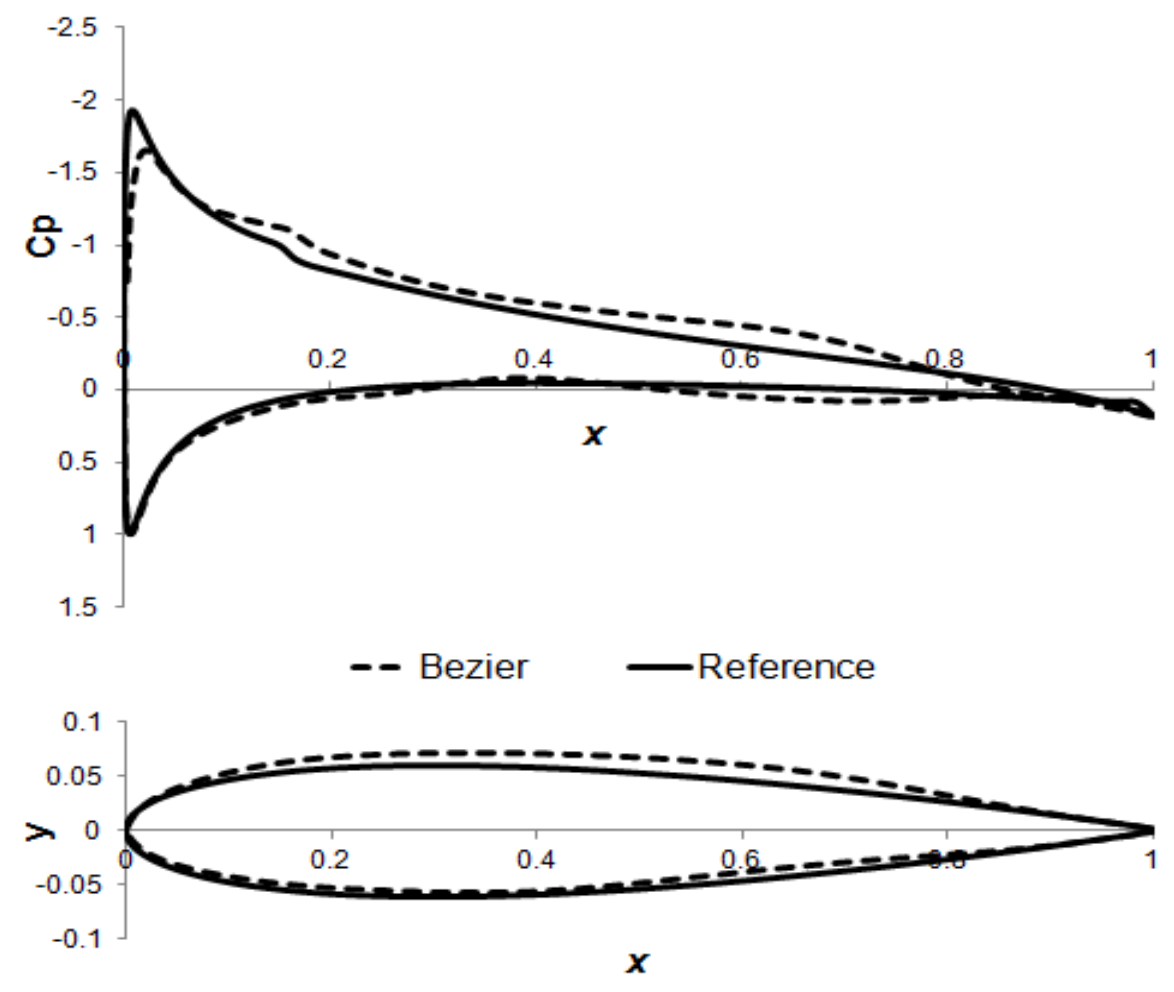

Fig 10 Optimum aerofoil geometry found through Bezier parameterization technique and corresponding Cp distribution compared with the reference
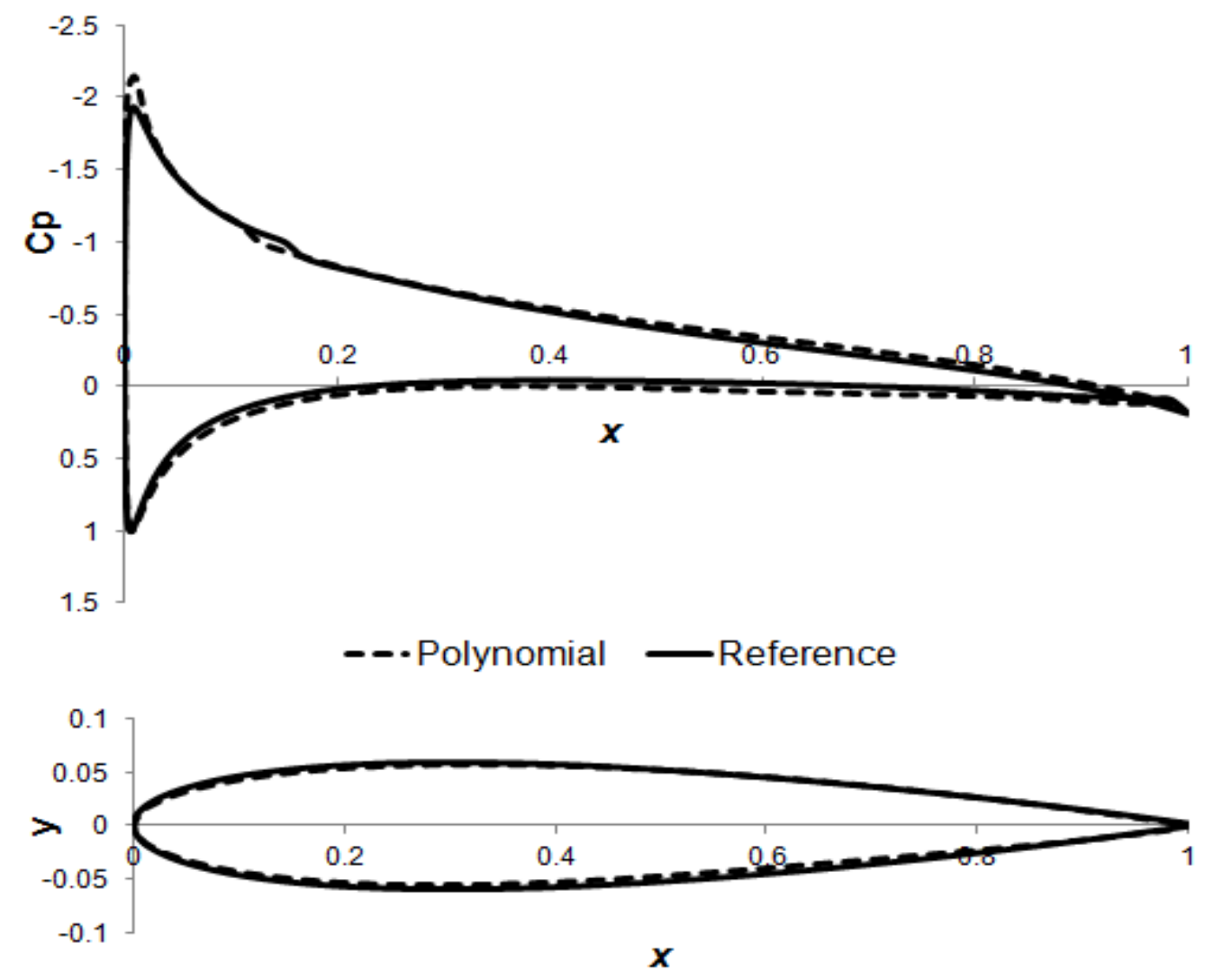

Fig 11 Optimum aerofoil geometry found through Polynomial parameterization technique and corresponding Cp distribution compared with the reference 